\title{
FURTHER RESULTS ON ELEMENTARY LIE ALGEBRAS AND LIE A-ALGEBRAS
}

\author{
David A. Towers \\ Department of Mathematics, Lancaster University \\ Lancaster LA1 4YF, England \\ and \\ Vicente R. Varea ${ }^{1}$ \\ Department of Mathematics, University of Zaragoza \\ Zaragoza, 50009 Spain
}

\begin{abstract}
A finite-dimensional Lie algebra $L$ over a field $F$ of characteristic zero is called elementary if each of its subalgebras has trivial Frattini ideal; it is an $A$-algebra if every nilpotent subalgebra is abelian. This paper is a continuation of the study of these algebras initiated by the authors in [10]. If we denote by $\mathcal{A}, \mathcal{G}, \mathcal{E}, \mathcal{L}, \Phi$ the classes of $A$-algebras, almost algebraic algebras, $E$-algebras, elementary algebras and $\phi$-free algebras respectively, then it is shown that:

$$
\mathcal{L} \subset \Phi \subset \mathcal{G}, \quad \mathcal{L} \subset \mathcal{A} \subset \mathcal{E} \text { and } \mathcal{G} \cap \mathcal{A}=\mathcal{L} .
$$

It is also shown that if $L$ is a semisimple Lie algebra all of whose minimal parabolic subalgebras are $\phi$-free then $L$ is an $A$-algebra, and hence elementary. This requires a number of quite delicate properties of parabolic subalgebras. Finally characterisations are given of $E$-algebras and of Lie algebras all of whose proper subalgebras are elementary.

Keywords: Lie algebra, elementary, $E$-algebra, $A$-algebra, almost algebraic, ad-semisimple, parabolic subalgebra.
\end{abstract}

\footnotetext{
${ }^{1}$ Supported by DGI Grant BFM2000-1049-C02-01
} 


\section{Introduction}

This paper is a continuation of the study initiated by the authors in [10]. Throughout $L$ will denote a finite-dimensional Lie algebra over a field $F$. The Frattini ideal of $L, \phi(L)$, is the largest ideal of $L$ contained in all maximal subalgebras of $L$. The Lie algebra $L$ is called $\phi$-free if $\phi(L)=0$, and elementary if $\phi(B)=0$ for every subalgebra $B$ of $L$. Lie algebras all of whose nilpotent subalgebras are abelian are called $A$-algebras; Lie algebras $L$ such that $\phi(B) \leq \phi(L)$ for all subalgebras $B$ of $L$ are called E-algebras. We are seeking to determine properties of, and inter-relationships between, these three classes of algebras. A linear Lie algebra $L \leq \operatorname{gl}(V)$ is almost algebraic if $L$ contains the nilpotent and semisimple Jordan components of its elements; an abstract Lie algebra $L$ is then called almost algebraic if $\operatorname{ad} L \leq \operatorname{gl}(L)$ is almost algebraic.

Throughout sections two and three $F$ is assumed to have characteristic zero. In section 2 we show first that $L$ is $\phi$-free if and only if it is almost algebraic and its nilradical is abelian. It follows from this that if $L$ is almost algebraic then $\phi(L)=N^{2}$, where $N$ is the nilradical of $L$. Zhao and $\mathrm{Lu}$ proved in [13] that every almost-algebraic $A$-algebra is elementary, whenever the ground field is algebraically closed of characteristic zero. We generalise this by showing that $L$ is an almost-algebraic $A$-algebra if and only if it is elementary (and without the assumption of an algebraically closed field). From this we deduce that if $L$ is a Lie $A$-algebra with a $\phi$-free solvable radical, then $L$ is elementary. The final result in this section is that if $L$ is a Lie $A$-algebra then it is an $E$-algebra.

A subalgebra $P$ of $L$ is called parabolic if $P \otimes_{F} \bar{F}$ contains a Borel subalgebra (that is, a maximal solvable subalgebra) of $L \otimes_{F} \bar{F}$, where $\bar{F}$ is the algebraic closure of $F$. The main purpose of section 3 is to prove two results. The first is that if $P$ is a minimal parabolic subalgebra of $L$ then the following are equivalent: $P$ is $\phi$-free; the nilradical of $P$ is abelian; and $P$ is elementary. The second is that if $L$ is a semisimple Lie algebra all of whose minimal parabolic subalgebras are $\phi$-free then $L$ is an $A$-algebra, and hence elementary. In order to establish these results we need a number of properties of parabolic subalgebras. Some of these may be known, but we know of no references to them other than that Völklein in [12] shows that if $L$ is semisimple then the minimal parabolic subalgebras of $L$ are the idealisers of the maximal nil subalgebras of $L$. He uses, however, the canonical correspondence between the connected subgroups of the identity component of the automorphism group of $L$ and the algebraic subalgebras of $L$. Our proofs are based entirely on internal properties of $L$ itself and so, 
we believe, are of interest in themselves. We also include these proofs for the convenience of the reader.

In the final section we look more closely at Lie $E$-algebras. In particular we give a characterisation of them over a field of characteristic zero. We also characterise Lie algebras all of whose proper subalgebras are elementary. These generalise Theorems 4.3 and 4.7 and Corollaries 4.4 and 4.5 of [9] by removing the requirement that the underlying field be algebraically closed.

We will denote vector space direct sums by $\oplus$ and semidirect products by $\rtimes$. If $A$ is a subalgebra of $B$ we will write $A \leq B$, whereas $A<B$ will mean that $A$ is a proper subalgebra of $B$. The (solvable) radical (resp. nilradical) of $L$ will be denoted by $R(L)$ (resp. $N(L)$ ), whilst $A \operatorname{soc}(L)$ will denote the sum (necessarily direct) of the minimal abelian ideals of $L$.

\section{Preliminary results}

First we reveal the relationship between almost-algebraic and $\phi$-free Lie algebras.

Proposition 2.1 Let L be a Lie algebra over a field of characteristic zero.

(i) If $L$ is $\phi$-free, then $L$ is almost algebraic.

(ii) Let $L$ be almost algebraic. Then $L$ is $\phi$-free if and only if its nilradical is abelian.

Proof. (i) Let $L$ be $\phi$-free. By [8] we have that $L=N(L)+S$ where $S$ is a subalgebra of $L$ such that $\operatorname{ad}_{L} S$ is completely reducible and $N(L)=$ $\operatorname{Asoc}(L)$. From [1, Theorem 2.2] it follows that $L$ is almost algebraic.

(ii) By $[1$, Theorem 2.2] we have that $L=N(L)+S$ where $S$ is a subalgebra of $L$ such that $\operatorname{ad}_{L} S$ is completely reducible. Now, assume that $N(L)$ is abelian. Then we have that $N(L)=$ Asoc $L$. So $L$ is $\phi$-free by [8]. The converse follows from [8].

Corollary 2.2 Let $L$ be an almost-algebraic Lie algebra over a field of characteristic zero. Then $\phi(L)=N^{2}$, where $N$ is the nilradical of $L$. 
Proof. Clearly $N^{2}$ is almost algebraic and so $L / N^{2}$ is almost algebraic, by [1, Lemma 4.1]. Moreover, $N\left(L / N^{2}\right)$ is abelian and hence $\phi$-free by Proposition 2.1. It follows that $\phi(L) \subseteq N^{2}$. The reverse inclusion is given by [8, Theorem $6.5]$.

If $B$ is a subalgebra of $L$ we define the idealiser of $B$ in $L$ to be $I_{L}(B)=$ $\{x \in L:[x, B] \leq B\}$. Next we show that a Lie algebra is an almost-algebraic $A$-algebra if and only if it is elementary, thereby generalising the result of Zhao and Lu proved in [13]. First we need the following result.

Proposition 2.3 Let $L$ be an almost-algebraic Lie algebra over a field of characteristic zero. If every almost-algebraic subalgebra of $L$ is $\phi$-free, then $L$ is elementary.

Proof. Let $B$ be a subalgebra of $L$. Then the idealiser of $B$ in $L, I_{L}(B)$, is almost algebraic, by [1, Theorem 2.3]. By our hypothesis, we have $\phi\left(I_{L}(B)\right)=0$. But now $B$ is an ideal of $I_{L}(B)$, so $\phi(B) \leq \phi\left(I_{L}(B)\right)$, by $[8$, Corollary 4.2$]$. Hence $L$ is elementary.

Theorem 2.4 Let L be a Lie algebra over a field of characteristic zero. Then $L$ is an almost-algebraic A-algebra if and only if it is elementary.

Proof. $(\Rightarrow)$ Let $L$ be an almost-algebraic $A$-algebra and let $B$ be an almostalgebraic subalgebra of $L$. Then by Corollary 2.2 it follows that $\phi(B)=N^{2}$, where $N$ is the nilradical of $B$. Therefore, $\phi(B)=0$ since $N$ is abelian. Hence $L$ is elementary by Proposition 2.3.

$(\Leftarrow)$ This follows from $[10$, Corollary 4.7$]$.

Corollary 2.5 Let L be a Lie A-algebra over a field of characteristic zero. If $R(L)$ is $\phi$-free, then $L$ is elementary.

Proof. Assume that $R(L)$ is $\phi$-free. By Proposition 2.1 we have that $R(L)$ is almost algebraic. Then, from [1, Corollary 3.1] it follows that $L$ is also almost algebraic. So, $L$ is elementary by Theorem 2.4 .

Corollary 2.6 Let $L$ be an almost-algebraic Lie A-algebra over a field of characteristic zero. Then $L$ splits over each of its ideals. 
Proof. This follows from Theorem 2.4 and [9, Lemma 2.3].

Finally we have that Lie $A$-algebras are necessarily $E$-algebras.

Proposition 2.7 Let $L$ be a Lie A-algebra over a field of characteristic zero. Then $L$ is an E-algebra.

Proof. We have that $L / \phi(L)$ is an $A$-algebra, by [6, Lemma 1]. On the other hand, we have that $L / \phi(L)$ is $\phi$-free and so almost algebraic by Proposition 2.1. Then $L / \phi(L)$ is elementary, by Theorem 2.4. So, $L$ is an $E$-algebra, by [11, Proposition 2].

Denote by $\mathcal{A}, \mathcal{G}, \mathcal{E}, \mathcal{L}, \Phi$ the classes of $A$-algebras, almost algebraic algebras, $E$-algebras, elementary algebras and $\phi$-free algebras respectively. Then, to summarise, what we have shown is the following:

$$
\mathcal{L} \subset \Phi \subset \mathcal{G}, \quad \mathcal{L} \subset \mathcal{A} \subset \mathcal{E} \text { and } \mathcal{G} \cap \mathcal{A}=\mathcal{L}
$$

\section{Parabolic subalgebras}

Throughout this section $L$ denotes a (non-zero) semisimple Lie algebra over a field $F$ of characteristic zero. We denote by $\bar{F}$ the algebraic closure of $F$, and write $\bar{S}=S \otimes_{F} \bar{F}$ for each subspace $S$ of $L$. For each subalgebra $S$ of $L$, let $U(S)$ denote the set of ad-nilpotent elements in the solvable radical, $R(S)$, of $S$. Our main objective in this section is to show that in order to check whether a Lie algebra is elementary it suffices to look at its minimal parabolic subalgebras. First we have some properties of the nilradical of a parabolic subalgebra.

Proposition 3.1 Let $P$ be a parabolic subalgebra of $L$. Then

(i) $N(P)$ is nil in $L$;

(ii) if $P<Q<L$, then $N(Q)<N(P)$; and

(iii) $P=I_{L}(N(P))$. 
Proof. Since $\overline{N(P)}$ is the nilradical of $\bar{P}$ and $\overline{I_{L}(N(P))}=I_{\bar{L}}(\overline{N(P)})$ (see [2, pages 42 and 36]) we may assume that $F$ is algebraically closed. Now $P$ is conjugate to a standard parabolic subalgebra and so we can assume it is of the following form. Let $H$ be a Cartan subalgebra of $L$ and let $\Delta$ be the set of roots corresponding to $H$. Then

$$
P=H \oplus \Sigma_{\alpha \in \Delta^{+}} L_{\alpha} \oplus \Sigma_{\alpha \in \Omega_{1}} L_{\alpha},
$$

where $\Omega_{1} \subseteq \Delta^{-}$. Let $\Omega_{1}^{\prime}=\left\{\alpha \in \Delta^{+} \mid-\alpha \notin \Omega_{1}\right\}$. Then we have that

$$
N(P)=\Sigma_{\alpha \in \Omega_{1}^{\prime}} L_{\alpha}
$$

It follows that $N(P)$ is nil in $L$ and (i) is proved.

To prove (ii), suppose that $P<Q<L$. Then $Q$ is also parabolic and so has the same form as $P$ but with $\Omega_{1}$ replaced by $\Omega_{2}$ where $\Omega_{1} \subset \Omega_{2}$. We have that $\Omega_{2}^{\prime} \subset \Omega_{1}^{\prime}$ and hence $N(Q)<N(P)$.

To prove (iii), put $Q=I_{L}(N(P))$ and suppose that $P<Q$. By (ii) we have $N(Q)<N(P)$. On the other hand, as $N(P)$ is a nilpotent ideal of $Q$ we have $N(P) \leq N(Q)$, which is a contradiction. Now the proof is complete.

The centre of $L$ is the set $Z(L)=\{x \in L:[x, L]=0\}$. A subalgebra $T$ of $L$ is said to be a toral subalgebra of $L$ if $T$ is abelian and $\operatorname{ad}_{L} t$ is semisimple for every $t \in T$. Next we need that if $P$ is an algebraic subalgebra of $L$ then $U(P)$ behaves well under field extension.

Lemma 3.2 Let $U$ be a nil subalgebra of $L$. Then $\bar{U}$ is also a nil subalgebra of $\bar{L}$

Proof. We have that $\bar{U}$ is a nilpotent algebraic subalgebra of $\bar{L}$, since these properties are inherited from $U$ (see [4, p.181]). So, $\bar{U}=U(\bar{U}) \oplus T$, where $T$ is a toral subalgebra of $\bar{L}$ and $[T, U(\bar{U})]=0$, by [3, Theorem 4]. We have $T \leq Z(\bar{U})=\overline{Z(U)}$ (see [2, page 36]). As $Z(U)$ consists of commuting ad-nilpotent elements, we have that $\overline{Z(U)}$ is a nil subalgebra of $\bar{L}$. This yields that $T$ is both nil and toral in $\bar{L}$ and so $T \leq Z(\bar{L})=0$. This yields that $\bar{U}=U(\bar{U})$ and so $\bar{U}$ is nil in $\bar{L}$.

Proposition 3.3 Let $P$ be an algebraic subalgebra of $L$. Then $\overline{U(P)}=$ $U(\bar{P})$ 
Proof. Since $P$ is algebraic, we have that $P=U(P) \oplus M$, where $M=$ $S \oplus Z(M)$ and $S$ is semisimple ( $M$ is a Levi factor of $P$ ), by [3, Theorem 4]. It follows that $\bar{P}=\overline{U(P)} \oplus \bar{M}$ and $\bar{M}=\bar{S} \oplus Z(\bar{M})$. On the other hand, from Lemma 3.2, it follows that $\overline{U(P)}$ is a nil ideal of $\bar{L}$. So, $\overline{U(P)} \leq U(\bar{P})$. This yields that $\overline{U(P)}=U(\bar{P})$.

The following results are concerned with relationships between parabolic subalgebras and certain nil subalgebras.

Lemma 3.4 Let $U$ be a nil subalgebra of $L$ and put $P=I_{L}(U)$. If $U=$ $U(P)$, then $P$ is parabolic.

Proof. This follows from Proposition 3.3 and [7, Theorem 29.8.1]

Proposition 3.5 Let $U$ be a nil subalgebra of $L$ and put $P=I_{L}(U)$. Then there is a parabolic subalgebra $Q$ of $L$ satisfying:

(i) $U \leq U(Q)$;

(ii) $P \leq Q$; and

(iii) $U(P) \leq U(Q)$.

Proof. Put $U_{0}=U$ and $Q_{1}=I_{L}\left(U_{0}\right)$. Define inductively the two sequences $\left\{U_{i}\right\}_{i \geq 0},\left\{Q_{i}\right\}_{i \geq 1}$ by $Q_{i}=I_{L}\left(U_{i-1}\right) U_{i}=U\left(Q_{i}\right)$. Then $Q_{i}=I_{L}\left(U_{i-1}\right)=$ $I_{L}\left(U\left(Q_{i-1}\right)\right) \geq Q_{i-1}$, so these sequences are increasing. This yields that there is an integer $j$ such that $Q_{j}=Q_{j+1}$; that is $Q_{j}=I_{L}\left(U_{j}\right)$. It follows from Lemma 3.4 that $Q=Q_{j}$ is a parabolic subalgebra of $L$. We have that $U \leq U_{j}=U(Q)$, giving (i), and $P=I_{L}(U)=Q_{1} \leq Q$, giving (ii).

Finally, $Q_{i}=I_{L}\left(U_{i-1}\right)$ so $U_{i-1}$ is a nil ideal of $Q_{i}$ and $U_{i-1} \leq U\left(Q_{i}\right)$, whence $U_{1}=U(P) \leq U(Q)$, giving (iii).

Proposition $3.6 \quad$ (i) If $U$ is a maximal nil subalgebra of $L$, then $I_{L}(U)$ is a minimal parabolic subalgebra of $L$.

(ii) If $P$ is a minimal parabolic subalgebra of $L$, then $U(P)$ is a maximal nil subalgebra of $L$ 
Proof. (i): Let $U$ be a maximal nil subalgebra of $L$. We have that $U$ is a nil ideal of $I_{L}(U)$ and so $U \leq U\left(I_{L}(U)\right.$. By the maximality of $U$, we must have that $U=U\left(I_{L}(U)\right)$. From Lemma 3.4 it follows that $I_{L}(U)$ is parabolic. Now let $Q$ be a parabolic subalgebra with $Q \leq I_{L}(U)$. By Proposition 3.1 we have that $U=N\left(I_{L}(U)\right) \leq N(Q)=U(Q)$. By the maximality of $U$ it follows that $U=N(Q)$. This yields that $I_{L}(U)=Q$ by Proposition 3.1 again, and so $I_{L}(U)$ is a minimal parabolic subalgebra of $L$.

(ii): Let $P$ be a minimal parabolic subalgebra of $L$. Suppose that there is a nil subalgebra $V$ of $L$ such that $U(P)<V$. By Engel's Theorem we have that $U(P)<V \cap I_{L}(U(P))$. As $P$ is parabolic, $I_{L}(U(P))=P$. Put $W=V \cap P$. Let $M$ be a Levi factor of $P$; so that $P=U(P) \oplus M$, $M=Z(M) \oplus S$ where $Z(M)$ is toral in $L$ and $S$ is a semisimple subalgebra of $L$.

We see that $W=U(P) \oplus(M \cap V)$. Since $Z(M)$ is toral it follows that $M \cap V=S \cap V$. So, $S \cap V$ is a non-trivial nil subalgebra of the semisimple Lie algebra $S$. From Proposition 3.5 there is a parabolic subalgebra $Q$ of $S, Q \neq S$, containing $S \cap V$. Let $B$ a Borel subalgebra of $\bar{S}$ contained in $\bar{Q}$. Then, we have that $R(\bar{P})+B$ is a maximal solvable subalgebra of $\bar{P}$. Since $\bar{P}$ is parabolic in $\bar{L}$, it follows that $R(\bar{P})+B$ is a Borel subalgebra of $\bar{L}$. We have $\overline{R(P)+Q}=R(\bar{P})+\bar{Q} \geq R(\bar{P})+B$. Therefore, $R(P)+Q$ is a parabolic subalgebra of $L$ contained in $P$, which contradicts the minimality of $P$. Hence $U(P)$ is a maximal nil subalgebra of $L$.

A Lie algebra $L$ is said to be ad-semisimple if ad $x$ is semisimple for every $x \in L$. Then we have the following criterion for a parabolic subalgebra to be minimal.

Corollary 3.7 $A$ parabolic subalgebra $P$ of $L$ is minimal if and only if $P / U(P)$ is ad-semisimple.

Proof. Let $M$ be a Levi factor of $P$. Let us first suppose that $P$ is minimal. Then, by Proposition 3.6 it follows that $U(P)$ is a maximal nil subalgebra of $L$ and so $M$ is ad-semisimple. Now assume that $P / U(P)$ is ad-semisimple, so that $M$ is ad-semisimple. As $Z(L)=0$, we see that $U(P)$ is a maximal nil subalgebra of $P$. Since $P=I_{L}(P)$, it follows from Engel's Theorem that $U(P)$ is maximal nil subalgebra of $L$.

We now have the results that we need to show the role played by the minimal parabolic subalgebras in determining whether or not $L$ is elementary. 
Lemma 3.8 Let $P$ be a minimal parabolic subalgebra of $L$. Then the following are equivalent

(i) $P$ is $\phi$-free;

(ii) $N(P)$ is abelian; and

(iii) $P$ is elementary.

Proof. (i) $\Rightarrow($ ii): Since $P$ is algebraic, it follows from Corollary 2.2 that $N(P)^{2}=\phi(P)=0$.

(ii) $\Rightarrow$ (iii): Since $N(R(P))=N(P)$ and $R(P)$ is algebraic, it follows from Proposition 2.1 that $R(P)$ is $\phi$-free. By [10, Theorem 2.5] we have that $R(P)$ is elementary. On the other hand, since $U(P) \leq R(P)$, it follows from Corollary 3.7 that $P / R(P)$ is ad-semisimple. But [10, Proposition 4.4] now implies that $P$ is elementary.

(iii) $\Rightarrow(\mathrm{i})$ : This is trivial.

Theorem 3.9 Let L be a semisimple Lie algebra over a field of characteristic zero, and suppose that all minimal parabolic subalgebras of $L$ are $\phi$-free. Then $L$ is an A-algebra, and hence elementary.

Proof. First we show that $L$ is an $A$-algebra. Let $U$ be a nilpotent subalgebra of $L$. Then $U$ is contained in a maximal solvable subalgebra $\Gamma$ of $L$. As $\Gamma$ is algebraic we can write $\Gamma=U(\Gamma) \dot{+} T$ where $T$ is a toral subalgebra. Let $N$ be a maximal nil subalgebra of $L$ containing $U(\Gamma)$, and let $P$ be the idealiser in $L$ of $N$. By Proposition 3.6, $P$ is minimal parabolic and hence $\phi$-free. It follows from Lemma 3.8 that $P$ is elementary, and hence an $A$-algebra by Proposition 2.4. This yields that $U(\Gamma)$ is abelian. But then $\Gamma$ is $\phi$-free and hence elementary, by [10, Theorem 2.5]. It follows that $U$ is abelian and that $L$ is an $A$-algebra.

By 2.4 it follows that $L$ is elementary.

\section{$4 \quad$ E-algebras}

First we have an easy strengthening of Corollary 2.2 of [10]. 
Proposition 4.1 Let $L$ be a solvable Lie algebra over a perfect field. Then $L$ is an E-algebra if and only if $L$ is strongly solvable.

Proof. This follows from [10, Corollary 2.2] and [11].

Next we have the following versions of Theorem 4.3 and Corollaries 4.4 and 4.5 of [9] with the assumption that the underlying field be algebraically closed removed.

Theorem 4.2 Let $L$ be a Lie algebra over a field of characteristic zero. Then $L$ is an E-algebra if and only if one of the following holds:

(i) L is solvable;

(ii) L is elementary and semisimple; or

(iii) $L=R \oplus S$, where $R=R(L)$ is the radical of $L, S=S_{1} \oplus S_{2}, S_{1}$ is an ad-semisimple ideal of $S, S_{2}$ is an elementary and semisimple ideal of $S$, and $S_{2} R \leq \phi(L)$.

Proof. $(\Rightarrow)$ : Let $L$ be an $E$-algebra and suppose that $L$ is not solvable or semisimple. By Levi's Theorem we can write $L=R \oplus S$ where $S$ is a semisimple subalgebra of $L$. If $U$ is a subalgebra of $L$ then write $\bar{U}$ for its image in $L / \phi(L)$ under the natural homomorphism. We have $\bar{L}=\bar{R} \oplus \bar{S}$ and $\bar{S} \cong S$ is elementary and semisimple. Put $S=S_{1} \oplus S_{2}$ where $S_{2}$ is the largest semisimple ideal of $L$. If $\overline{S_{1}} \neq 0$, then $\overline{S_{1}} \cong S_{1}$ is ad-semisimple, as in [10, Theorem 4.6]. Clearly $S_{2} R \leq \phi(L)$, so (iii) holds.

$(\Leftarrow)$ : If (i) holds then $L$ is strongly solvable (since the ground field has characteristic 0 ) and the result follows from Proposition 4.1 above. If (ii) holds the result is clear. So suppose that (iii) holds. Then $\bar{L}=\overline{L_{1}} \oplus \overline{S_{2}}$ where $\overline{L_{1}}=\bar{R} \oplus \overline{S_{1}}$. Now $\overline{L_{1}} / \bar{R} \cong \overline{S_{1}} \cong S_{1}$ is ad-semisimple and $\bar{R}$ is elementary, so $\overline{L_{1}}$ is elementary, by [10, Proposition 4.4]. It follows that $\bar{L}$ is elementary, and hence that $L$ is an $E$-algebra.

Corollary 4.3 Let L be a Lie algebra over a field of characteristic zero, and suppose that the radical of $L$ is nilpotent. Then Lis an E-algebra if and only if one of the following holds:

(i) L is nilpotent; 
(ii) L is elementary and semisimple; or

(iii) $L=\left(R \oplus S_{1}\right) \oplus S_{2}$, where $R=R(L)$ is the radical of $L, S_{1}$ is an adsemisimple subalgebra of $L$, and $S_{2}$ is an elementary and semisimple ideal of $L$.

Proof. This follows as in [9, Corollary 4.4].

Corollary 4.4 Let $L$ be a perfect Lie algebra (i.e., $L=L^{2}$ ) over a field of characteristic zero. Then $L$ is an E-algebra if and only if $L$ is elementary and semisimple.

Finally we consider non-elementary Lie algebras all of whose proper subalgebras are elementary. We call such algebras minimal non-elementary Lie algebras. The following extends Theorem 4.7 of [9].

Theorem 4.5 Let $L$ be a Lie algebra over a field of characteristic zero. Then $L$ is a minimal non-elementary Lie algebra if and only if

(i) $L=L^{2} \rtimes F x$, where $L^{2}$ is abelian and $0 \neq \phi(L)=$ AsocL is the biggest ideal of $L$ properly contained in $L^{2}$, or

(ii) $L$ is the three-dimensional Heisenberg algebra.

Proof. $(\Rightarrow)$ First note that $L$ must be an $E$-algebra. Suppose that $L$ is not solvable. Then $R=R(L)$ is elementary and so almost algebraic by [10, Proposition 4.1]. Hence $L$ is almost algebraic, by [1, Corollary 3.1]. Moreover, the nilradical is elementary and so abelian. It follows that $L$ is $\phi$-free, by Proposition 2.1, and so elementary - a contradiction. This yields that $L$ is solvable.

Suppose that $L$ is not nilpotent. Then $\phi(L) \neq L^{2}$ (see, for example, $[8$, section 5]), so there is a maximal subalgebra $M$ of $L$ such that $L=L^{2}+M$. Choosing $B$ to be a subalgebra minimal with respect to the property that $L=L^{2}+B$ we have $L^{2} \cap B \leq \phi(B)=0$ (see [8, Lemma 7.1]), so $L=L^{2} \oplus B$ and $B$ is abelian. Moreover, $L^{2}$ is nilpotent and elementary, and so abelian.

Suppose that $\operatorname{dim} B>1$. Let $K$ be a maximal subalgebra of $B$. Then $M=L^{2}+K$ is a maximal subalgebra of $L$, so $M$ is elementary and hence $\phi$-free. It follows from [8, Theorem 7.4] that $L^{2}$ is completely reducible as 
a $K$ - module, and hence that each element of $K$ acts semisimply on $L^{2}$ ([5, Theorem 10, page 81]). But every element of $B$ is contained in a maximal subalgebra of $B$ and so acts semisimply on $L^{2}$. This yields that $L^{2}$ is a completely reducible $B$-module, whence $L^{2} \leq$ Asoc $L$ and $L$ splits over Asoc $L$. But then $\phi(L)=0$, by [8, Theorem 7.3], and $L$ is elementary, a contradiction. Thus $\operatorname{dim} B=1$, so put $B=F x$.

So we now have $L=L^{2} \rtimes F x$ where $L^{2}$ is abelian. Let $C$ be an ideal of $L$ with Asoc $L<C<L^{2}$ and put $D=C+F x$. Then $D \neq L$ so $\phi(D)=0$, giving Asoc $D=N(D) \geq C$. But every minimal ideal of $D$ is inside $L^{2}$ and invariant under ad $x$ and so is an ideal of $L$. It follows that $C=\operatorname{Asoc} D \leq$ Asoc $L$, a contradiction. Hence Asoc $L$ is the biggest ideal of $L$ properly contained in $L^{2}$. We must have Asoc $L \leq \phi(L)$, since otherwise $L$ splits over Asoc $L$, as in paragraph two above. As $\phi(L) \neq L^{2}$ this means that $\phi(L)=$ Asoc $L$ and we have case (i).

Suppose now that $L$ is nilpotent. Then $L$ is not abelian, so $\operatorname{dim} L \geq 3$ and $L$ has a chain of ideals

$$
0=L_{0}<L_{1}<\ldots<L_{n}=L,
$$

where $\operatorname{dim} L_{i}=i$ and $L L_{i} \leq L_{i-1}$ for $1 \leq i \leq n$. Let $L_{1}=F z, L_{2}=F y+F z$, and let $x$ be any element of $L$. If $F x+F y+F z$ is abelian for every $x \in L$ then $L$ is abelian, a contradiction. Hence $L$ has a subalgebra isomorphic to the three-dimensional Heisenberg algebra. Such a subalgebra cannot be proper as it is not elementary.

$(\Leftarrow)$ It is clear that the three-dimensional Heisenberg algebra is minimal non-elementary, so assume that $L$ is as described in (i). It suffices to show that the maximal subalgebras of $L$ are elementary. Let $M$ be a maximal subalgebra of $L$. Since Asoc $L \leq M$ either $M=L^{2}$ or $M=$ Asoc $L+F x$ for some $x \in L \backslash L^{2}$. In the former case $M$ is abelian and so elementary. So assume that $M=\operatorname{Asoc} L+F x$ and $L=L^{2}+F x$. Let $A$ be a minimal ideal of $M$. Then $A \leq$ Asoc $L$ and $[A, L] \leq[A, x] \leq A$ so $A$ is an ideal of $L$. It follows that Asoc $M=$ Asoc $L$ and $M$ splits over Asoc $M$. Hence $M$ is $\phi$-free. Since $M$ is clearly an $E$-algebra, it is elementary.

Note: The algebras $L$ described in Theorem 4.5 (i) are $A$-algebras (as every nilpotent subalgebra of $L$ is inside $L^{2}$ ) that are not elementary. They are therefore not almost algebraic. 


\section{References}

[1] L. Auslander and J. Brezin, 'Almost Algebraic Lie Algebras', $J$. Algebra 8 (1968), 295-313.

[2] N. Bourbaki, 'Elements of Mathematics, Lie Groups and Lie Algebras, Chapters 1-3', Springer (1989).

[3] C. Chevalley, 'Algebraic Lie Algebras', Ann. Math (2) 48 (1947), 91100.

[4] C. Chevalley, 'Théorie des Groupes de Lie,' Hermann, Paris, 1968.

[5] N.JAcobson, 'Lie algebras', Dover, New York, (1979).

[6] A.A. Premet And K.N. Semenov, 'Varieties of residually finite Lie algebras', Math. USSR Sbornik 65 (1990), 109-118.

[7] P. Tauvel and R.W.T. Yu, 'Lie Algebras and Algebraic Groups', Springer, Berlin/Heidelberg/New York (2005).

[8] D.A. Towers, 'A Frattini theory for algebras', Proc. London Math. Soc. (3) 27 (1973), 440-462.

[9] D.A. Towers, 'Elementary Lie algebras', J. London Math. Soc. (2) 7 (1973), 295-302.

[10] David A. Towers and Vicente R. Varea, 'Elementary Lie Algebras and Lie A-algebras', J. Algebra 312 (2007), 891-901.

[11] E.L. Stitzinger, 'Frattini subalgebras of a class of solvable Lie algebras', Pacific J. Math. 34 (1970), 177-182.

[12] Helmut Völklein, 'Lattice Isomorphisms of Lie Algebras and Algebraic Groups', J. Algebra 107 (1987), 82-97.

[13] X.C. ZhaO And C.H. Lu, 'An equivalent description of elementary Lie algebras (Chinese)', J. Math. Study 36 (2003), 202-210. 